\title{
IDENTIFYING THE DIFFERENCES IN THE CAUSAL FACTORS OF TRUCK-INVOLVED CRASHES IN RURAL AND URBAN AREAS
}

\author{
DONGHYUNG YOOK ${ }^{1 *}, \mathrm{JUN}^{2} \mathrm{LEE}^{2} \&$ SUNNIE HAAM ${ }^{3}$ \\ ${ }^{1}$ Korea Research Institute for Human Settlements, Republic of Korea \\ ${ }^{2}$ Korea Transport Institute, Republic of Korea \\ ${ }^{3}$ University of Seoul, Republic of Korea
}

\begin{abstract}
Various factors such as speed, variability of speed, traffic flows and the proportion of trucks affect the probability of truck-involved crashes. Numerous attempts have been made to identify the causal factors of truck-involved crashes, such as traffic volume, speed characteristics and geometric characteristics. Most of the research focused on identifying the causal factors or establishing models to represent the relationship between crashes and the identified factors. However, few studies have compared the differences in the impact of a coefficient by the type of region. This study aims to analyse the differences in the causal factors of truckinvolved crashes in rural and urban areas. The applicability of the count models is examined owing to the low number of trucks involved in the crashes. The models for each area type are established using zero-inflated Poisson regression and negative binomial regression model for rural and urban areas, respectively. Our results indicate that sight distance is the single factor responsible for truck-involved crashes in rural areas, whereas annual average daily traffic, shoulder width and alignment are the contributors to truck-involved crashes in urban areas.

Keywords: count model, Poisson regression, truck-involved crashes, zero inflated regression.
\end{abstract}

\section{INTRODUCTION}

Various factors affect the probability and consequences of a crash. Over the past several years, considerable efforts have been made in the search for causal factors and in exploring the relationship among these causal factors. The findings of many studies conducted to investigate the causal factors of vehicle crashes enable us to categorize these factors into three types: driver, vehicle and operating environmental factors. Each category includes the following details:

Vehicle characteristics:

- Vehicle configuration [1];

- Size and weight [2];

- Vehicle design [3];

- Mechanical condition [4].

*ORCID: https://orcid.org/0000-0001-9634-3232 
Operating environment:

- Traffic characteristics [5];

- Adverse weather [6];

- Roadway characteristics $[7,8]$.

Driver related:

- Fatigue [9];

- Alcohol [10].

Most vehicle crashes can be linked to either one factor or a combination of these factors. However, special attention needs to be paid to the trucks involved in the crashes owing to the uniqueness of their characteristics.

The manoeuvrability of trucks is significantly different from that of other vehicle types. Trucks can be significantly heavier than other vehicles in the traffic. They are less manoeuvrable, start more slowly, and take a longer time to stop [10,11]. The driver seats in trucks are much higher than those in passenger vehicles; therefore, truck drivers can see much further down the road. In addition, trucks are mostly used in work settings as part of a job. Various relationships among the causal factors that characterise the truck-involved crashes have been identified.

Craft [12] observed that in addition to vehicle characteristics, the roadway is a factor that is linked to $14 \%$ of trucks and $16 \%$ of other vehicles involved in two type crashes. In terms of the operational environment, many studies have attempted to identify the causal factors such as the traffic volume, speed characteristics and geometric characteristics to distinguish the countermeasures of truck-involved crashes from those of other types of crashes. However, most of the studies focused on identifying the causal factors or establishing models to determine the relationship between the crashes and the identified factors. Furthermore, few studies have compared the differences in the impact of the causal factors according to the region. If the dominant causal factor in a specific type of roadway or region can be identified, effective and cost-efficient countermeasures can be implemented on the roadway, and better informed decisions on transportation safety policy for truck drivers can be made. For instance, if speed is determined to be the more dominant factor in truck-involved crashes in urban areas than in rural areas, the safety of the roadway in urban areas can be improved by implementing countermeasures different from those implemented in rural areas.

The purpose of this study is to investigate the differences in the causal factors of truckinvolved crashes between rural and urban areas. In Section 2, an overview of truck-involved crashes is presented. Section 3 presents a review of studies on various causal factors affecting truck-involved crashes. We also analyse the relationship between the causal factors and truck-involved crashes by the type of area (rural and urban areas), as presented in Section 4.

\section{OVERVIEW OF TRUCK-INVOLVED CRASHES}

In general, the consequences of a crash are more severe when a truck is involved than when other types of vehicles are involved [13]. Approximately 5,200 trucks were associated with fatal traffic accidents in the U.S. each year from 2001 to 2005; this number is larger than that of truck-involved crashes from 1998 to 2002 by a factor of more than 200 per year $[14,15]$. The proportion of large trucks involved in fatal crashes (11.2\%) was the highest compared with that of other vehicle types, whereas other crash types and injury and property damage 
Table 1: Police-reported motor vehicle traffic crashes in 2006.

\begin{tabular}{llll}
\hline Crash type & Large trucks $(A)$ & All vehicles $(B)$ & Percentage $(A / B) \times 100$ \\
\hline Fatal & $4,321(1.2)$ & $38,588(0.6)$ & 11.2 \\
Injury & $77,000(20.9)$ & $1,746,000(29.2)$ & 4.4 \\
Property damage only & $287,000(78.0)$ & $4,189,000(70.1)$ & 6.9 \\
Total & $368,000(100)$ & $5,974,000(100)$ & 6.2 \\
\hline
\end{tabular}

only (PDO) crashes comprised $4.4 \%$ and $6.9 \%$ of all vehicle cases, respectively. This means that large truck crashes have the highest risk of fatality. The characteristics of a truck, such as its heavy weight and slow manoeuvrability, can contribute to the unusual high record of fatal crashes.

Moreover, when a fatal accident involving a truck occurs, the probability of having victims of the fatal accident from the other vehicle is significantly higher than that of victims from the truck. In $2005,74.5 \%$ of the total fatalities of truck-involved accidents were the occupants of other vehicles. However, the fatality rate of the occupants of trucks is approximately $15 \%$. The study by Khorashadi et al. also reported similar findings [16]. They noted that approximately 4,700 people are killed nationwide in accidents involving large trucks and approximately $85 \%$ of the fatalities are the occupants of non-truck vehicles. Data gathered over several years show a steady increase in this phenomenon.

The spatial distribution of truck-involved fatal crashes corresponds to the level of exposure of truck traffic to possible crashes. General Accounting Office [17] reported that rural roads account for approximately $40 \%$ of the total vehicle mile travelled (VMT); however, more than $60 \%$ of all fatalities occur on rural roads. Truck crashes follow the same pattern; over $60 \%$ of the total fatal crashes occurred in rural areas in 2005, whereas $35.1 \%$ occurred in urban areas. The level of exposure of truck traffic seems to have a dominant effect on truck-involved crashes, but it can be proved that it is not. Additional causal factors of truckinvolved crashes are discussed and compared through systematic analysis in this paper.

\section{LITERATURE REVIEW}

Most studies on the causal factors of truck-involved crashes focused on the characteristics of the driver, working conditions, and size of the truck companies. The ease of data accessibility, ease of data quantification and recognisability of the causal relationship are possible reasons for the extensive research on the relationship between the characteristics of the driver or working conditions and truck-involved crashes. In addition, many studies also focused on the operating environment under which truck-involved crashes occur.

According to previous studies, truck-involved crashes are mainly influenced by the sociodemographic and behavioural characteristics of the drivers. It is known that socio-demographic factors such as gender and age have different effects on truck-involved crashes. Male drivers are less likely to experience severe crashes than female drivers [18]. It is known that older truck drivers are more involved in severe crashes than younger drivers [19]. In terms of behavioural characteristics, as one might expect, speeding and driving under influence are among the most influential factors that contribute to truck-involved crashes with severe injuries $[16,18,20]$. The severity of truck-involved crashes varies depending on the driving environment. It is easy to guess that the severity of truck-involved crashes under snow, fog and icy conditions can be high. In addition to weather conditions, the effects of types of roads are 
also different. It is known that the severity of truck-involved crashes in interstate highways is much higher than that in local street crashes [21].

However, only few studies [22,23] have compared the differences in the impact of the causal factors according to the type of area. The traffic characteristics in rural and urban areas are different and can be used to distinguish the causal factors of truck-involved crashes based on the type of area [24]. This section reviews two studies that investigated the differences in the causal factors of truck-involved crashes based on the type of area.

Khorashadi et al. [16] investigated the differences in the scale and direction of the impacts of explanatory variables when considering urban and rural large truck crash occurrences. Using a multinomial logit model, the authors computed the impact of various causal factors on the severity of the injury of the driver using four years of California accident data. A comparison of the estimated results for the models revealed notable differences and similarities, and variables that obtained the statistical significance in both the urban and rural models varied significantly in terms of their impact on the outcome of the severity of the injury of the driver.

Unlike the above study, Golob [25] focused on the relationship between traffic conditions and truck-involved crashes using crash data obtained from records of accidents on six freeways in Orange County, California for over 2 years. The study found that the truck traffic mix has a positive impact on the increasing trend of trucks involved in crashes, whereas the number of lanes and section traffic volume per lane have a negative impact on truck crashes. In addition, the temporal distribution of truck-related crashes and the impact of the number of lanes were evaluated in the study. Table 2 presents the estimated model coefficients.

Table 2: Logit model specification of truck involvement by Golob (2004).

\begin{tabular}{lll}
\hline Independent variable & Coefficient & $t$-Statistic \\
\hline Percentage of section AADT that is truck & 0.106 & 7.63 \\
Percentage of section truck traffic that is 5+ axles & 0.027 & 10.565 \\
Number of lanes on accident side of freeway & -0.075 & -2.354 \\
Section traffic volume (AADT) per lane (106) & -8.087 & -3.025 \\
Time period 12 AM to 2:59 AM weekdays & 0.747 & 4.321 \\
Time period 3 AM to 6:59 AM weekdays & 0.92 & 8.992 \\
Time period 7 AM to 7:59 AM weekdays & 0.611 & 5.583 \\
Time period 8 AM to 8:59 AM weekdays & 0.702 & 6.592 \\
Time period 9 AM to 1:59 PM weekdays & 1.196 & 15.806 \\
Time period 2 PM to 3:59 PM weekdays & 0.642 & 7.188 \\
Time period 4 PM to 4:59 PM weekdays & 0.392 & 3.461 \\
Day is a Saturday & -0.311 & -2.712 \\
Day is a Sunday & -0.601 & -4.058 \\
Road surface is wet or slippery & -0.352 & -3.384 \\
Constant & -3.063 & -9.382 \\
\hline
\end{tabular}

Sources : Golob [25]. 


\section{CASE STUDY}

The two studies reviewed in the previous section examined the impact of various causal factors on truck-involved crashes. The first study analysed the different impacts of the causal factors of truck-involved crashes by the area type. However, the impacts of some independent variables could not be easily compared owing to the different selection of independent variables in the urban and rural models. Further, some general causal factors of truck-involved crashes were not considered in the model. The second study investigated the relationship between causal factors and truck-involved crashes by applying appropriate forms of variables. However, a method of comparing urban and rural models, which distinguishes the impact of the causal factors by the type of area, is lacking.

The crash data used in this study were obtained in the period 2001-2006 from selected high-risk locations on multi-lane highways in the state of Virginia, US. The crash data were obtained from FR300s, the Police Crash Report from the state of Virginia, US.

\subsection{Brief description of the crash data}

The total number of non-truck-involved crashes observed in the period in rural and urban areas was 3,951 and 11,104, respectively, whereas the number of truck-involved crashes in rural and urban areas was 77 and 183, respectively. The distributions of the number of crashes by speed, truck percentage and annual average daily traffic (AADT) are similar for nontruck-involved crashes and truck-involved crashes within one area type. However, the distribution of the number of crashes differs in urban and rural areas. For instance, when the speed is between 55 and $60 \mathrm{mph}$, approximately $60 \%$ of crashes occurred in rural areas and $13 \%$ in urban areas. It should be noted that an increase in the number of crashes does not signify its dominant impact on crashes. Instead, it could be related to an increase in traffic exposure.

\subsection{Methodology}

In this study, we applied different models including Poisson, negative binomial regression [26] and zero-inflated regression models. The description of zero-inflated regression models [27], [28] begins by pointing out the limitations of the Poisson model.

The Poisson regression model explicitly considers the nonnegative integer-value of the dependent count variable.

The probability of an event count $y_{i}$ for a given vector of $x_{i}$ is given by the Poisson distribution:

$$
P\left(Y_{i}=y_{i} \mid x_{i}\right)=\frac{e^{-\mu_{i}} \mu_{i}^{y_{i}}}{y_{i} !}, y_{i}=0,1,2,
$$

where, $\mu_{i}$ is the conditional mean.

The Poisson regression model assumes that the data are equally dispersed, which implies that the conditional variance should be equal to the conditional mean. However, the conditional variance rarely equals the conditional mean in real-life data, which are often overdispersed, with the variance exceeding the mean.

The motivation for zero-inflated models is designed to reflect real-life data that frequently shows overdispersion and excess zeros. Zero-inflated count models enable the modelling of the excess zeros while allowing for overdispersion, which assumes two types of individuals producing zeros; (1) Those who produce a zero count with a probability of 1 (group 1) and (2) 


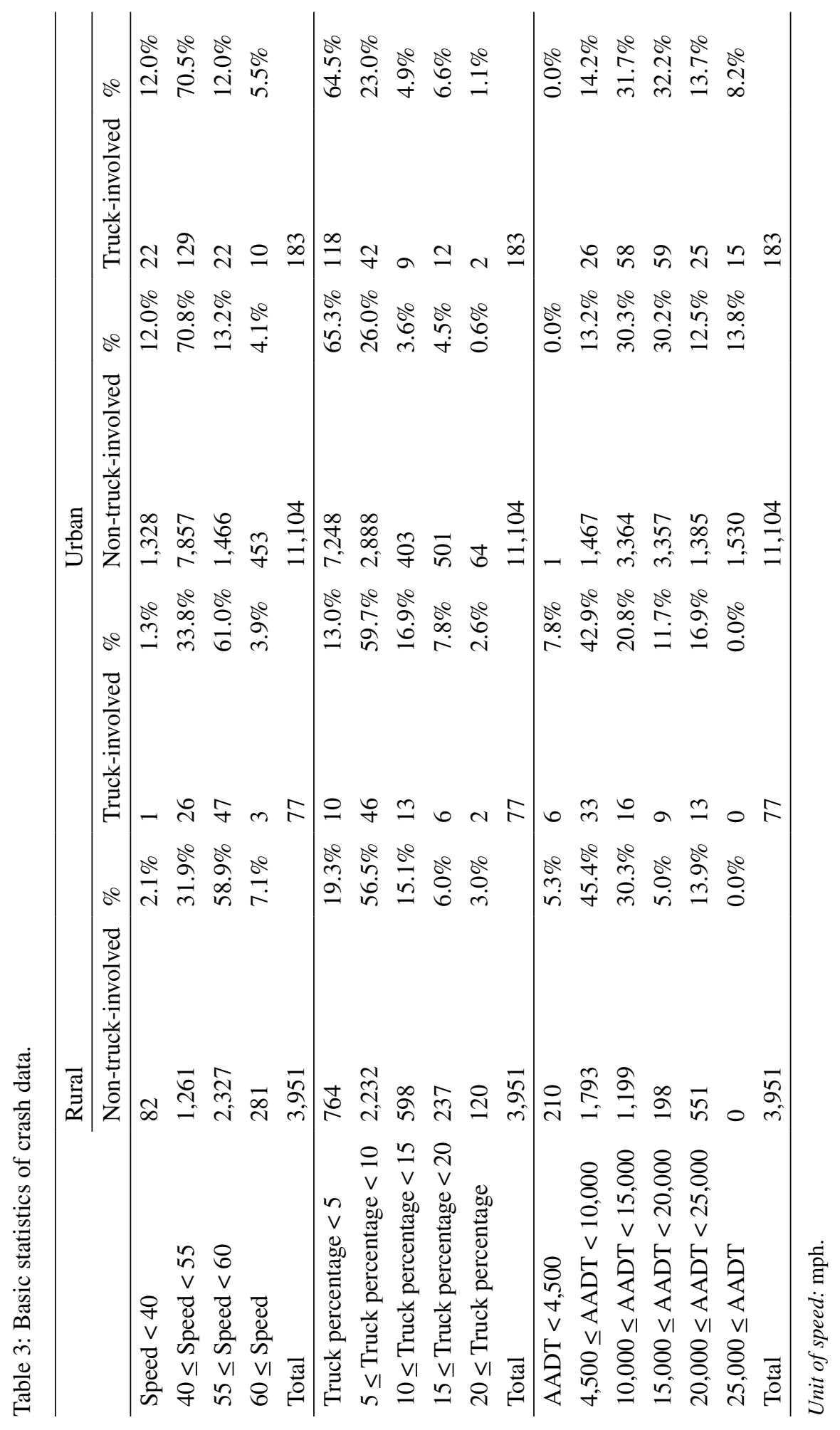


those who have counts predicted by the standard Poisson (group 2). When 'zero' is observed it could be from either of two groups.

The probability of zero from group (1):

$$
P\left(Y_{i}=0 \mid x_{i}, h_{i}\right)=\tau_{i} \times 1=\tau_{i},
$$

where $\tau_{i}=G\left(k_{i}, \theta\right), k_{i}$ refers to the vector of zero-inflated covariates, and $\theta$ is corresponding coefficients.

Thus, probability of zero from group (2) is as follows:

$$
P\left(Y_{i}=0 \mid x_{i}, h_{i}\right)=\left(1-\tau_{i}\right) \times \frac{e^{-\mu_{i}} \mu_{i}^{0}}{0 !}=\left(1-\tau_{i}\right) e^{-\mu_{i}}
$$

The probability of non-zero from group (2) is as follows:

$$
P\left(Y_{i}=y_{i} \mid x_{i}, h_{i}\right)=\left(1-\tau_{i}\right) \times \frac{e^{-\mu_{i}} \mu_{i}^{y_{i}}}{y_{i} !}
$$

Finally, the probability of $\left\{Y_{i}=y_{i} \mid x_{i}, h_{i}\right\}$ is given by:

$$
P\left(Y_{i}=y_{i} \mid x_{i}, h_{i}\right)=\left\{\begin{array}{cc}
\tau_{i}+\left(1-\tau_{i}\right) e^{-\mu_{i}} & \text { if } y_{i}=0 \\
\left(1-\tau_{i}\right) \times \frac{e^{-\mu_{i}} \mu_{i}^{y_{i}}}{y_{i} !} & \text { if } y_{i}>0
\end{array}\right.
$$

The function that relates the $x_{i}, h_{i}$ to the probability $\tau_{i}$ is known as the zero-inflated link function, and it can be specified as either a logistic function or a probit function.

\subsection{Model specifications}

The data of locations of the crashes are merged into the corresponding site data comprising information of the geometric and traffic characteristics of the sites. The following attributes comprise the geometric information merged with the traffic characteristics for each site:

- Speed limit;

- Alignment;

- Shoulder width;

- Sight distance;

- AADT;

- 85th percentile speed (operational speed);

- Truck percentage.

In the model specifications, these attributes act as independent variables, whereas the number of accidents involving trucks is the dependent variable. All the count models introduced are examined to determine how well they represent the observed data based on the type of area. The best model specification is determined by evaluating the performance measures, such as the 
$p$-value for the model specification and $t$-statistics for the coefficients. The model specification is used to determine the best model for each area type, and the associated model coefficients are identified. Tables 6 and 7 present the model specifications for the rural and urban models. For the rural model, the zero-inflated model exhibits the best performance for fitting the observed data, whereas the negative binomial model represents the data from urban areas.

\subsection{Results}

According to the final model, sight distance seems to be an important factor that determines the number of crashes involving trucks in the rural model. Because the sight distance has been taken by log, we can interpret the coefficient for sight distance that a unit change $(\mathrm{km})$ in the sight distance reduces crashes by around 8.22 in the rural area. However, insufficient data on the number of crashes involving trucks limits the significance of the model. The $p$-value of the model is not desirable.

The urban model is well represented by the negative binomial model. The model specification measures have desirable values. Three attributes, namely AADT, alignment, and shoulder width, were determined to be the significant factors affecting truck-involved crashes in urban areas. The model indicates that AADT increases the number of truck-involved crashes. This result agrees with the interpretation of AADT from other causal factor studies. Because the AADT indicates the level of exposure of traffic to possible accidents, it generally has a positive sign as a causal factor of crashes. An increase in the alignment and shoulder width seems to decrease the number of truck-involved crashes. This means that failure to guide a vehicle within lanes could be due to insufficient shoulder width. This is important for trucks. The large width of trucks needs sufficient lane width, particularly in urban areas where lane width is sometimes compromised due to the need to realize more lanes. Because the driving performance of trucks is sensitive to the grade of the road, alignment has a greater impact than that of the shoulder width.

Table 4: Rural model specification: zero inflated Poisson model.

\begin{tabular}{llllll}
\hline Model part & Coefficients & Estimate & $p$-Value & Signif. & Chisq Pr (> Chisq) \\
\hline \multirow{2}{*}{ Count model } & (Intercept) & 16.1053 & $1.25 \mathrm{E}-02$ & 0.05 & \\
& Sight distance & -2.1067 & 0.00321 & 0.01 & \multirow{2}{*}{0.1429} \\
\multirow{2}{*}{ Zero model } & (Intercept) & 28.842 & $3.10 \mathrm{E}-03$ & 0.01 & \\
& Sight distance & -3.191 & 0.00502 & 0.01 & \\
\hline
\end{tabular}

Table 5: Urban model specification: negative binomial model.

\begin{tabular}{lllll}
\hline Coefficients & Estimate & $P$-value & Signif. & Chisq Pr (> Chisq) \\
\hline (Intercept) & -6.63484 & 0.0002 & 0.001 & \\
AADT & 0.38362 & 0.03933 & 0.05 & \multirow{2}{*}{$0.0094 * *$} \\
Alignment (\%) & -0.11997 & 0.0685 & 0.1 & \\
Shoulder width & -0.04183 & 0.04869 & 0.05 & \\
\hline
\end{tabular}

Signif. codes: '**', 0.01 , the dispersion parameter is 1.3108 . 
The model specification results indicate that completely different attributes affect truckinvolved crashes in each area type. According to the results, ensuring sufficient sight distance is the most important task for transportation safety engineers in rural areas. This implies that a location with a short sight distance in rural areas might be a hot spot for truck-involved crashes. A road section with a short sight distance could be curved, and truck drivers need to drive carefully. Countermeasures for preventing truck-involved crashes in urban areas should focus on managing low AADT as well as ensuring a sufficient shoulder width and stable alignment.

\section{SUMMARY AND CONCLUSIONS}

This study investigated the different causal factors of truck-involved crashes in urban and rural areas. Fundamental differences between the two types of crashes (truck-involved crashes and other types of crashes) were identified through descriptive statistics of truckinvolved crashes. The difference in the severity of truck-involved crashes, the distribution of victims according to demographic factors, and the difference in fatal accidents according to the type of area were also identified.

Various count models such as Poisson regression, negative binomial regression and zeroinflated Poisson regression were examined to determine the best model representing the observed data for the two area types. The best-fitted models for each area type indicate that the causal factors of truck-involved crashes are different for different area types. For rural areas, which are represented using the zero-inflated Poisson regression model, sight distance was determined as the single contributor to truck-involved crashes. For urban areas, a few attributes including the shoulder width, AADT, and alignment were identified as contributors to truck-involved crashes.

These conclusions contribute to policy making to prevent truck-involved crashes. For example, first, the conclusions imply that the method of preventing truck-involved crashes between rural and urban areas should be approached fundamentally differently. Second, in rural areas, road sections where sight distance is compromised have a high probability of truck accidents. To avoid crashes, the roadways must be designed such that the sight distance is not compromised. In the case of urban areas, it has been shown that truck accidents can be reduced by installing a dedicated lane that guides only truck traffic.

\section{REFERENCES}

[1] Mihan, M., Mahdi, R., Khaled, K., An investigation of influential factors of downgrade truck crashes: a logistic regression approach. Journal of Traffic and Transportation Engineering, 6(2), pp. 185-195, 2019.

[2] Zou, W, Wang, X, Zhang, D., Truck crash severity in New York city: an investigation of the spatial and the time of day effects. Accident Analysis \& Prevention, 99, pp. 249261, 2017.

[3] Muhammad, T.H., Milan, Z., Khaled, K., Investigating occupant injury severity of truck-involved crashes based on vehicle types on a mountainous freeway: a hierarchical Bayesian random intercept approach. Accident Analysis \& Prevention, 144, pp. 105654, 2020.

[4] Blower, D., Green P. E., Matteson A., Condition of trucks and truck crash involvement: evidence from the large truck crash causation study. Transportation Research Record: Journal of the Transportation Research Board, 2194(1), pp. 21-28, 2010.

[5] Ali, B., Al-Bdairi, N.S.S., Determinant of injury severities in large truck crashes: a weekly instability analysis. Safety Science, 131, pp. 104911, 2020. 
[6] Bhaven, N., Tung, L.W., Shanshan, Z et al., Weather impacts on single-vehicle truck crash injury severity. Journal of Safety Research, 58, pp. 57-65, 2016.

[7] Mouyid, B.I., Salvador, H., Modeling Injury outcomes of crashes involving heavy vehicles on Texas highways. Transportation Research Record, 2388, pp. 28-36, 2013.

[8] Dong, C., David, B.C., Stephen, H.R. et al., Differences in passenger car and large truck involved crash frequencies at urban signalized intersections: an exploratory analysis. Accident Analysis \& Prevention, 62, pp. 87-94, 2014.

[9] Mohamed, M.A., Rebecca, F., Khaled, K. et al., Effects of truck traffic on crash injury severity on rural highways in Wyoming using Bayesian binary logit models. Accident Analysis \& Prevention, 117, pp. 106-113, 2018.

[10] David, E.C., Thomas, M.C., Curtis, M.G. et al., A driver focused truck crash prediction model. Transportation Research Part E: Logistics and Transportation Review, 46, pp. 683-692, 2010.

[11] Hyun, K., Jeong, K.S., Tok, A. et al., Assessing crash risk considering vehicle interactions with trucks using point detector data. Accident Analysis \& Prevention, 130, pp. 75-83, 2019.

[12] Craft, R., Federal motor carrier safety administration research and technology: today and tomorrow. Proceedings of the 84th Annual Meeting of the Transportation Research Board. Washington, DC, 2005.

[13] Milhan, M., Mahdi, R., Mustaffa, N.R., Khaled, K., Predicting injury severity and crash frequency: insights into the impacts of geometric variables on downgrade crashes in Wyoming. Journal of Traffic and Transportation Engineering, 7, pp. 375-383, 2020.

[14] Matteson, A., Jarossi, L., Woodrooffe, J., Trucks Involved in Fatal Accidents Factbook 2002, The University of Michigan, Transportation Research Institute: Ann Arbor, MI, 2004.

[15] Matteson A., Jarossi L., Woodrooffe J., Trucks involved in Fatal Accidents Factbook 2005, The University of Michigan, Transportation Research Institute: Ann Arbor, MI, 2008.

[16] Khorashadi, A., Niemeier, D., Shankar, V., et al., Differences in rural and urban driverinjury severities in accidents involving large-trucks: an exploratory analysis. Accident Analysis \& Prevention, 37, pp. 910-921, 2005.

[17] US General Accounting Office (GAO), Report to Congressional Committees, Federal and State Efforts to Address Rural Road Safety Challenges, Washington, DC: GAO, May 2004 (GAO-04-663).

[18] Uddin, M., Huynh, N., Truck-involved crashes injury severity analysis for different lighting conditions on rural and urban roadways. Accident Analysis \& Prevention, 108, pp. 44-55, 2017.

[19] Zhu, X., Srinivasan, S., A comprehensive analysis of factors influencing the injury severity of large-truck crashes. Accident Analysis \& Prevention, 43, pp. 49-57, 2011.

[20] Lemp, J.D., Kockelman, K.M., Unnikrishnan, A., Analysis of large truck crash severity using heteroskedastic ordered probit models. Accident Analysis \& Prevention, 43, pp. 370-380, 2011.

[21] Ahmed, M.M., Franke, R., Ksaibati, K., Shinstine, D.S., Effects of truck traffic on crash injury severity on rural highways in Wyoming using Bayesian binary logit models. Accident Analysis \& Prevention, 117, pp. 106-113, 2018.

[22] W. Horrey, C. Wickens Multiple resource modeling of task interference in vehicle control, hazard awareness and in-vehicle task performance. Proceedings of the Second 
International Driving Symposium on Human Factors in Driver Assessment, Training and Vehicle Design, Park City, UT (2003).

[23] J. Lee, F. Mannering Impact of roadside features on the frequency and severity of runoff-roadway accidents: an empirical analysis. Accident Analysis \& Prevention, 34(2), pp. 149-161, 2002.

[24] Nabeel Saleem, S.A.B., Salvador, H., Comparison of contributing factors for injury severity of large truck drivers in run-off-road crashes on rural and urban roadways: accounting for unobserved heterogeneity. International Journal of Transportation Science and Technology, 9, pp. 116-127, 2020.

[25] Golob, F.T., Regan, A.C., Traffic Conditions and Truck Accidents on Urban Freeways. University of California, Institute of Transportation Studies: Irvine, CA, 2004.

[26] Greene, W. H. Accounting for excess zeros and sample selection in Poisson and negative binomial regression models, NYU Working Paper, No. EC-94-10. New York, NY, 1994.

[27] Cohen, A. C. Estimation in mixtures of discrete distributions. Proceedings of the International Symposium on Discrete Distributions, Montreal, 373-378, 1963.

[28] Lambert, D. Zero-inflated Poisson regression, with an application to defects in manufacturing, Technometrics, 34, pp. 1-14, 1992. 